\title{
Constructing universal graphs for induced-hereditary graph properties
}

\author{
Izak Broere* \\ Department of Mathematics, University of Johannesburg \\ izakb@uj.ac.za \\ Johannes Heidema \\ Department of Mathematical Sciences, University of South Africa \\ johannes.heidema@gmail.com \\ Peter Mihók \\ Department of Applied Mathematics, Technical University of Košice \\ peter.mihok@tuke.sk
}

\begin{abstract}
Rado constructed a (simple) denumerable graph $R$ with the positive integers as vertex set with the following edges: For given $m$ and $n$ with $m<n, m$ is adjacent to $n$ if $n$ has a 1 in the $m$ 'th position of its binary expansion. It is well known that $R$ is a universal graph in the set $\mathcal{I}_{c}$ of all countable graphs (since every graph in $\mathcal{I}_{c}$ is isomorphic to an induced subgraph of $R$ ).

In this paper we construct graphs which are universal in or for $\mathcal{P}$ for different inducedhereditary properties $\mathcal{P}$ of countable graphs. Constructions of universal graphs for the graph properties containing all graphs with colouring-number at most $k+1$ and $k$-degenerate graphs are obtained by restricting the edges of $R$. Results on the properties of these graphs are given and relationships between them are explored. This is followed by a general recursive construction which proves the existence of a countable universal graph for any induced-hereditary property of countable general graphs. A general construction of universal graphs for products of properties of graphs is also presented. The paper is concluded by a comparison between the two types of constructions of universal graphs.
\end{abstract}

AMS Subject Classification (from MSC2010): $05 C 63$

Keywords: countable graph, universal graph, induced-hereditary graph property, graph with colouring-number $k+1, k$-degenerate graph, product of graph properties

\section{Introduction}

For general graph theoretic notions, the notation and terminology of [8] will be used. In particular, for any two graphs $G$ and $H=\left(V^{\prime}, E^{\prime}\right)$, we say that $G$ is a subgraph of $H$, denoted by $G \subseteq H$, if there is a subset $V \subseteq V^{\prime}$ and a subset $E \subseteq E^{\prime}$ such that $(V, E)$ is a graph which is isomorphic to $G$; $G$ is an induced subgraph of $H$, denoted by $G \leq H$, if $G$ is isomorphic to such a graph $(V, E)$ of which $E$ contains all the edges $x y \in E^{\prime}$ for which $x, y \in V$. We shall also write $G \subset H(G<H)$ to denote the fact that $G$ is a subgraph (an induced subgraph respectively) of $H$ which is not isomorphic to $H$.

There is (up to isomorphism) clearly only one subgraph induced by a given subset $W$ of the vertex set $V$ of a graph $G=(V, E)$; this subgraph is denoted by $G[W]$ and called the subgraph

\footnotetext{
*Present address: Department of Mathematics and Applied Mathematics, University of Pretoria; izak.broere@up.ac.za
} 
of $G$ generated (or spanned) by $W$.

All graphs considered here are countable graphs; we assume the vertex set of a denumerable graph to be $\mathbf{N}=\{1,2, \ldots\}$ unless stated otherwise. The nature of the vertices, being positive integers, will play a crucial role in the constructions of the graphs to be considered in Sections 2.2 and 2.3 .

For notions related to hereditary graph properties the notation and terminology of [2] will be used. For ease of reference we formulate some of the basic definitions in this paper too. A (graph) property is an isomorphism-closed subclass of the class of all graphs. Since we have, in a graph property, no reason to distinguish between isomorphic copies of a graph, we consider the class of all (simple) graphs to be a set and we use the notation $\mathcal{I}_{c}$ to denote this set of (countable) graphs. Two (disjoint) subsets of any property $\mathcal{P}$ of countable graphs $\left(\mathcal{P}=\mathcal{P}_{c}\right)$ are also important for us and we introduce notation for them too: $\mathcal{P}_{f}$ will denote the set of finite graphs in $\mathcal{P}$ and $\mathcal{P}_{d}$ will denote the set of denumerable graphs in $\mathcal{P}$. In this paper we will often have occasion to deal with two graphs that are isomorphic and, if they are, we shall refer to any one of them as a clone of the other.

The symbol $\mathcal{E}$ will denote the empty property, i.e., the subset of $\mathcal{I}_{c}$ containing no graphs. The properties $\mathcal{I}_{c}$ and $\mathcal{E}$ are called trivial (properties).

A component of a graph is a $\leq$-maximal connected induced subgraph of that graph. A property $\mathcal{P}$ is called additive if for each graph $G$ all of whose components are in $\mathcal{P}$ we have that $G \in \mathcal{P}$ too. This is equivalent to saying that $\mathcal{P}$ is closed under taking disjoint unions of graphs. Suppose $\preceq$ is a partial order on the set $\mathcal{I}_{c}$. A property $\mathcal{P}$ is said to be $\preceq$-hereditary if, whenever $G \in \mathcal{P}$ and $H \preceq G$, then $H \in \mathcal{P}$ too. In particular, a property $\mathcal{P}$ is induced-hereditary if it is $\leq$-hereditary with respect to the relation $\leq$ to be an induced subgraph and $\mathcal{P}$ is hereditary if it is $\subseteq$-hereditary with respect to the relation $\subseteq$ to be a subgraph.

Let $\mathcal{P}$ be a set of countable graphs. Following [8], we define a graph $U$ to be a universal graph for $\mathcal{P}$ if every graph in $\mathcal{P}$ is an induced subgraph of $U$; it is a universal graph in $\mathcal{P}$ if $U \in \mathcal{P}$ too. Since a universal graph $U$ for $\mathcal{P}$ is allowed to be outside $\mathcal{P}$ and hence, presumably, to be uncountable, the existence of at least one such $U$ becomes trivial: take $U$ to be the disjoint union of one clone from each isomorphism class in $\mathcal{P}$ (i.e. of a "skeleton" of $\mathcal{P}$ ). The fact that this $U$ (which exudes an aura of lazy brute force) will in general be uncountable follows from the next lemma; in Section 3 we shall construct a countable $U$ for any induced-hereditary graph property $\mathcal{P}$.

Consider countable linear forests, i.e., graphs with a countable vertex set which have no cycles and in which every vertex is of degree at most two. Note that every component of such a linear forest is a finite path (with no or with two vertices of degree one) or an infinite path (with no or with one vertex of degree one). Also note that if two such linear forests are isomorphic, then they have the same number of components of each length, and that linear forests constitute an induced-hereditary graph property.

Lemma 1 There are uncountably many, in fact at least $2^{\aleph_{0}}$, pairwise non-isomorphic countable linear forests in which every component is a finite path.

Proof:

In order to prove this statement, we construct an injection $f$ from the real numbers in the interval $(0,1)$ into the set of all such graphs. Hence let $x \in(0,1)$ and suppose $x_{1} x_{2} \ldots$ is the decimal expansion of $x$. Then we define the linear forest $f(x)$ by letting, for each positive integer $k$, the number of components of order $k$ in $f(x)$ be $x_{k}$. By the remark immediately before the lemma we then have that, if $x, y \in(0,1)$ with $x \neq y$, then $f(x) \neq f(y)$, i.e., $f$ is an injection.

From Lemma 1 now follows that most graph properties contain uncountably many pairwise nonisomorphic graphs. Indeed, if a graph property $\mathcal{P}$ is hereditary or additive and contains, for 
every $k \in \mathbf{N}$, the path on $k$ vertices (and many do), then all the linear forests used in this lemma are in $\mathcal{P}$ and hence $\mathcal{P}$ is uncountable. So, for many (i.a. induced-hereditary) properties $\mathcal{P}$, the universal graph for $\mathcal{P}$ obtained by taking the disjoint union of a skeleton of $\mathcal{P}$ will not be countable - and has, of course, uncountably many components. Therefore the construction of a countable (usually denumerable) universal graph for $\mathcal{P}$ is of interest.

Rado [18] constructed the following (simple) denumerable graph on $\mathbf{N}$ : For given $m$ and $n$ with $m<n, m$ is adjacent to $n$ if $n$ has a 1 in the $m$ 'th position of its binary expansion. We shall denote this graph by $R$. It is well known that $R$ is a universal graph in the induced-hereditary property $\mathcal{I}_{c}$ of countable graphs. A very useful and, in fact, a characteristic property of $R$ is that it has the extension property: For every two finite disjoint sets $U$ and $V$ of vertices of $R$ there is a vertex not in $U \cup V$ which is adjacent to every vertex of $U$ and to no vertex of $V$.

In Section 2 we discuss examples and constructions of universal graphs for (and in) some well-known induced-hereditary properties of simple graphs and develop some properties of these universal graphs. In Section 3, dealing with general graphs, i.e., no longer simple graphs, a different type of construction delivers countable universal graphs for all induced-hereditary properties at one fell swoop. We compare and contrast features of the constructions in Sections 2 and 3 in the last Section 4.

\section{Universal graphs for induced-hereditary properties}

\subsection{Background}

The following table summarises some of the published results for some induced-hereditary properties of countable graphs. (There is more on (induced-)hereditary properties in [2].) Throughout this table, $k$ is a positive integer.

\begin{tabular}{|c|c|c|c|c|}
\hline Property & Description & $U \in \mathcal{P}_{d} ?$ & Characterisation of $U ?$ & Reference(s) \\
\hline $\mathcal{I}_{c}$ & All graphs & $\begin{array}{l}\text { Yes, the Rado } \\
\text { graph } R \in \mathcal{I}_{c}\end{array}$ & $\begin{array}{l}C \cong R \text { iff } C \text { has the } \\
\text { extension property }\end{array}$ & {$[18]$} \\
\hline $\mathcal{P}_{\text {fin }}$ & $\begin{array}{l}\text { Graphs with } \\
\text { all vertices of } \\
\text { finite degree }\end{array}$ & $\begin{array}{c}\text { Does not } \\
\text { exist in } \mathcal{P}_{\text {fin }}\end{array}$ & & $\begin{array}{l}\text { [18] (accredited } \\
\text { to N.G. de Bruijn) }\end{array}$ \\
\hline $\mathcal{L}_{k}$ & $\begin{array}{l}\text { Directed label- } \\
\text { led graphs }\end{array}$ & $\begin{array}{l}\text { Yes, the graph } \\
\quad L_{k} \in \mathcal{L}_{k}\end{array}$ & $\begin{array}{l}C \cong L_{k} \text { iff } C \text { has the } \\
k \text {-extension property }\end{array}$ & {$[4]$} \\
\hline$-\left\{K_{k+2}\right\}$ & $\begin{array}{c}K_{k+2} \text {-free } \\
\text { graphs }\end{array}$ & $\begin{array}{c}\text { Yes, the graph } \\
G_{k} \in-\left\{K_{k+2}\right\}\end{array}$ & $\begin{array}{l}C C \cong G_{k} \text { iff } C \text { has an } \\
\text { adapted extension property }\end{array}$ & [10] and [13] \\
\hline$-\left\{K_{m, n}\right\}$ & $\begin{array}{c}K_{m, n} \text {-free } \\
\text { graphs }\end{array}$ & $\begin{array}{l}\text { Exists if and only } \\
\text { if } m=1 \text { and } n \leq 3\end{array}$ & & {$[14]$} \\
\hline$-\left\{C_{3}\right\}$ & $\begin{array}{l}C_{3} \text {-free } \\
\text { graphs }\end{array}$ & $\begin{array}{c}\text { Yes, the graph } \\
G_{1} \in-\left\{C_{3}\right\}\end{array}$ & $\begin{array}{c}\text { Same as } K_{3^{-}} \\
\text {free graphs above }\end{array}$ & \\
\hline$-\left\{C_{4}\right\}$ & $\begin{array}{l}C_{4} \text {-free } \\
\text { graphs }\end{array}$ & $\begin{array}{l}\text { Does not exist } \\
\quad \text { in }-\left\{C_{4}\right\}\end{array}$ & & {$[12]$} \\
\hline $\begin{array}{c}-\left\{C_{n}\right\} \\
n \geq 5\end{array}$ & $\begin{array}{l}C_{n} \text {-free } \\
\text { graphs }\end{array}$ & $\begin{array}{l}\text { Does not exist } \\
\quad \text { in }-\left\{C_{n}\right\}\end{array}$ & & {$[6]$} \\
\hline$-\mathcal{S}$ & $\begin{array}{l}\text { Limited cycle- } \\
\text { free graphs }\end{array}$ & $\begin{array}{c}\text { Exists in }-\mathcal{S} \text { if } \\
\text { and only if } \mathcal{S}=\mathcal{S}_{k}\end{array}$ & & {$[7]$} \\
\hline$\rightarrow H$ & $\begin{array}{l}\text { Hom-property } \\
\text { for finite } H\end{array}$ & $\begin{array}{l}\text { Known to exist } \\
\text { in } \rightarrow H\end{array}$ & & {$[17]$} \\
\hline
\end{tabular}


In this table, $\mathcal{S}$ denotes a finite set of cycles and $\mathcal{S}_{k}$ denotes the set of odd cycles $\left\{C_{3}, C_{5}, \ldots, C_{2 k+1}\right\}$. Furthermore, for a given finite graph $H$ and a given set of connected finite graphs $\mathcal{T}$, the (additive and) induced-hereditary graph properties $\rightarrow H$ and $-\mathcal{T}$ are defined by

$\rightarrow H=\left\{G \in \mathcal{I}_{f}:\right.$ there is a homomorphism from $G$ into $\left.H\right\}$

$-\mathcal{T}=\left\{G \in \mathcal{I}_{f}:\right.$ for each $T \in \mathcal{T}, T$ is not an induced subgraph of $\left.G\right\}$.

In [11] it is also shown that there is no universal graph in any set $\operatorname{Forb}(G)$ of countable graphs obtained by taking a finite, 2-connected graph $G$ which is not complete and requiring that the graphs in $\operatorname{Forb}(G)$ are exactly those not containing $G$ as a subgraph.

In Section 3 we shall construct a countable universal graph for every induced-hereditary property of countable graphs (even without restricting us to simple graphs); this result includes each of the properties in the above table and many of the well-known properties mentioned in $[2]$.

\subsection{A universal graph in graphs with colouring-number at most $k+1$}

Definition 1 [9] For a given cardinal number $\alpha$, we say that a countable graph $G$ has colouringnumber $\alpha$ if the vertices of $G$ can be labelled as $v_{1}, v_{2}, \ldots$ in such a way that for each positive integer $\ell \geq 1$ the degree of $v_{\ell}$ in the subgraph of $G$ induced by $\left\{v_{1}, v_{2}, \ldots, v_{\ell}\right\}$ is less than $\alpha$, and $\alpha$ is the least cardinal number with this property.

In our use of this concept we will restrict ourselves to a finite cardinal (usually by taking $\alpha=k+1$ where $k$ is a positive integer). But our definition contains a further restriction when compared to the one given by Erdös and Hajnal in [9]: Our insistence on the existence of a labelling in the definition above is more restrictive than their requirement that the set of vertices has some well-ordering with certain properties (since ours corresponds to the least ordinal with the same properties).

Definition 2 The set of countable graphs with colouring-number at most $k+1$ is denoted by $\mathcal{C}_{k}$, i.e.,

$$
\mathcal{C}_{k}=\left\{G \in \mathcal{I}_{c}: \text { the colouring-number of } G \text { is at most } k+1\right\} ;
$$

it is an induced-hereditary property of graphs.

It is easy to prove (by an inductive argument using the labelling of the vertices of such a graph) that each graph with colouring-number $k+1$ has chromatic number at most $k+1$, explaining its naming. We shall discuss the relation between this concept and the concept of $k$-degenerate graphs in the next subsection. Erdös and Hajnal prove in [9] for a graph $G$ that, if the colouringnumber of every finite subgraph of $G$ is at most $k+1$, then $G$ has colouring-number at most $2 k$. They also provide an example which shows that this result is best possible, i.e., for every $k \geq 1$ there is a graph of which every finite subgraph has colouring-number at most $k+1$ but which does not have colouring-number $2 k-1$ or less.

We now construct a universal graph in $\mathcal{C}_{k}$ and discuss its properties. We denote this graph by $E_{k}$ and remark that it is obtained by keeping all the vertices but restricting the choice of edges of the Rado graph $R$. A corresponding construction of a universal graph for the property of $k$-degenerate graphs and a discussion of its properties is contained in the next subsection. Throughout these subsections, we assume that $k$ is a given positive integer. In the (finite) power series $n=\sum_{i=0}^{\infty} n_{i} 2^{i}$ we shall refer to $n_{i-1}(i \geq 1)$ as the entry in the $i$ 'th position of the binary expansion of the positive integer $n ; n_{i-1} \in\{0,1\}$.

Definition 3 The denumerable (simple) graph $E_{k}$ on $N$ has the following edges: For given positive integers $m$ and $n$ with $m<n, m$ is adjacent to $n$ if $n$ has at most $k+1$ ones in its binary expansion and has, in particular, a one in position $m$ and a one in position $x$ for some 


\section{$x>m$ of its binary expansion.}

The role of the one in position $x$ will become clear in the proofs of the results to follow.

It is easy to see that the sequence of subgraph relations $E_{1} \subseteq E_{2} \subseteq \cdots \subseteq R$ holds. However, no two of these graphs are isomorphic, as can be deduced from the following lemma.

Lemma 2 Let $k$ be a positive integer. Then
1. $K_{k+1} \subseteq E_{k}$
2. $K_{k+2} \not \subseteq E_{k}$
3. $K_{\infty} \subseteq R$
4. $K_{\infty} \nsubseteq E_{k}$

\section{Proof:}

1. Consider the following recursive construction of a clone of the complete graph $K_{k+1}$ in $E_{k}$ : Take an arbitrary vertex $m_{1} \geq 3$ of $E_{k}$. Construct $m_{2}$ as the vertex with a one only in positions $m_{1}$ and $m_{1}+1$ of its binary expansion. Then $m_{1}<m_{2}$ and the two are adjacent in $E_{k}$. More generally: If $m_{1}, m_{2}, \ldots, m_{i-1}$ are already constructed, define $m_{i}$ to be that integer with ones precisely in positions $m_{1}, m_{2}, \ldots, m_{i-1}$ and $m_{i-1}+1$ of its binary expansion; and stop the construction after the construction of $m_{k+1}$. It is then clear that the choices of the $m_{i-1}+1$ 's can play the role of the $x$ required in the definition of $E_{k}$ to ensure that each $m_{i}$ is adjacent to each of the previously constructed vertices, i.e., $\left\{m_{1}, m_{2}, \ldots, m_{k+1}\right\}$ induces a subgraph of $E_{k}$ which is a clone of $K_{k+1}$.

2. If $K_{k+2} \subseteq E_{k}$, say with $\left\{m_{1}, m_{2}, \ldots, m_{k+2}\right\}$ as vertex set with $m_{1}<m_{2}<\cdots<m_{k+2}$, then $m_{k+2}$ has at least $k+2$ ones in its binary expansion.

3. $R$ is a universal graph for, in fact in, the set of countable graphs.

4. This follows immediately from 2 . above.

For $E_{1}$ one can also see that if $E_{1}$ contains a cycle, then the largest vertex $n$ of this cycle is adjacent to two smaller vertices on this cycle. There is, however, no vertex $n$ in $E_{1}$ which is adjacent to both $\ell$ and $m$ if these two vertices satisfy $n>\ell, m$. Hence $E_{1}$ contains no cycles, i.e., $E_{1}$ is a forest. Also, every vertex of each $E_{k}$ is of infinite degree; in fact, for each $k \geq 2$, every $k$ vertices of $E_{k}$ have infinitely many common neighbours.

In our next two results we describe some properties of the set of graphs $\mathcal{C}_{k}$ and the graph $E_{k}$.

Theorem 1 Let $k$ be a positive integer. Then $E_{k}$ is a universal graph in $\mathcal{C}_{k}$ and has colouringnumber $k+1$.

\section{Proof:}

We first prove the inclusion $E_{k} \in \mathcal{C}_{k}$ : Consider the labelling of the vertices of $E_{k}$ given by their natural well-ordering as positive integers, i.e., let $v_{m}=m$ for each positive integer $m$. Then, for each $\ell \geq 2$, each vertex of the subgraph induced by $\left\{v_{1}, v_{2}, \ldots, v_{\ell}\right\}$ which is adjacent to $v_{\ell}$ corresponds to a one in the binary expansion of $v_{\ell}$. But, if there is at least one such adjacency, then $v_{\ell}$ has at most $k+1$ ones in its binary expansion of which at most $k$ correspond to vertices adjacent to $v_{\ell}$; hence the degree of $v_{\ell}$ is at most $k$ in that subgraph. Hence $E_{k} \in \mathcal{C}_{k}$. That $E_{k}$ has colouring-number $k+1$, no less, no more, follows from its definition, its universality in $\mathcal{C}_{k}$, and Lemma 2 parts 1 . and 2.

Next we shall prove by complete induction on the cardinality of its vertex set that every (finite or denumerable) graph $G$ with colouring-number at most $k+1$ is an induced subgraph of $E_{k}$. This is clearly true for a graph with only one vertex; assume that it is true for all graphs with colouring-number at most $k+1$ with at most $p-1$ vertices and let $G$ be a graph with colouring-number at most $k+1$ with $p$ vertices. Consider the vertex $v$ of $G$ with the largest label index and suppose it is of degree $\ell$ in $G$. Then $\ell \leq k$ and $G-v$ is a graph with colouring-number 
at most $k+1$ with $p-1$ vertices. Hence $G-v$ is an induced subgraph of $E_{k}$; assume that $m_{1}, m_{2}, \ldots, m_{\ell}$ are the vertices of $E_{k}$ corresponding to the $\ell$ neighbours of $v$ in $G$. Then we construct a number $n$ by choosing $\ell+1$ ones in its binary expansion in positions $m_{1}, m_{2}, \ldots, m_{\ell}$ and in some position $x$ which is large enough to ensure that $x>m_{i}$ for each $i$, that $n>m_{i}$ for each $i$, and that $n$ is not a vertex of $E_{k}$ corresponding to any vertex of $G-v$; there are zeros in all the other positions of the binary expansion of $n$. Clearly, this vertex $n$ can correspond to $v$ in an isomorphism between $G$ and an induced subgraph of $E_{k}$, completing the induction step.

Corollary 1 Let $k$ be a positive integer. Then

1. $E_{1}<E_{2}<\cdots<R$.

2. If $C$ is universal for $\mathcal{C}_{k}$ then $E_{k} \leq C$.

3. If $C$ is universal in $\mathcal{C}_{k}$ then $C \leq E_{k}$.

\section{Proof:}

1. It follows immediately from our definition of graphs with colouring-number $k+1$ that $\mathcal{C}_{k-1} \subseteq \mathcal{C}_{k}$ for every $k \geq 2$. Hence the sequence of relations $E_{1}<E_{2}<E_{3}<\cdots$ follow by Theorem 1 and the remark (already made) that no isomorphism holds between any two of them. Finally, $E_{k}<R$ is true for each $k$ since $R$ is a universal graph for $\mathcal{I}_{c}$ and $E_{k}$ is not isomorphic to $R$, by 3 . and 4 . of Lemma 2.

2. If $C$ is universal for $\mathcal{C}_{k}$, then $E_{k} \leq C$ by Theorem 1 .

3. If $C$ is universal in $\mathcal{C}_{k}$, then $C$ has colouring-number $k+1$ too, hence $C \leq E_{k}$ by Theorem 1 .

Note that the labelling $v_{m}$ for the vertex $m$ of $E_{k}$ used in the proof of Theorem 1 adds no value whatsoever. Henceforth, when working with $E_{k}$ as a graph with colouring-number $k+1$, we shall use the name $m$ of a vertex instead of some label $v_{m}$ and thus consider the labelling function to be the identity function.

We remark that $E_{k}$, unlike $R$, is not self-complementary since $E_{k}$ contains arbitrary large edgeless subgraphs so that $\overline{E_{k}}$ contains arbitrary large complete subgraphs, which is incompatible with having colouring-number $k+1$. There are, however, some properties of $E_{k}$ which are similar to properties of the Rado graph $R$; we now bring two of these into focus.

Definition 4 We say that a graph $C$ has the k-adjoining property if for every two finite disjoint sets $U$ and $V$ of vertices of $C$ with $|U| \leq k$ there is a vertex not in $U \cup V$ which is adjacent to every vertex of $U$ and to no vertex of $V$.

The graph $E_{1}$ is not homogeneous and, for $k \geq 2, E_{k}$ does not seem to be homogeneous (in the sense defined in [13]), i.e., not every isomorphism between two finite, isomorphic, induced subgraphs of $E_{k}$ can be extended to an automorphism of $E_{k}$. It does, however, possess a lesser property which we now define.

Definition 5 We say that a graph $C$ allows iso-extensions if $C$ is denumerable and every isomorphism between two finite induced subgraphs of $C$ has an extension to an isomorphism between two (not necessarily different) denumerable induced subgraphs of $C$.

Note that every denumerable homogeneous graph allows iso-extensions, but not conversely.

Lemma 3 The graph $E_{k}$ has the following properties:

1. $E_{k}$ has the k-adjoining property.

2. For any two finite, isomorphic, induced subgraphs $F$ and $G$ of $E_{k}$ with vertex sets $X$ and $Y$ respectively, any vertex $m$ of $E_{k}$ not in $F$ which is larger than each vertex of $F$ to which it is adjacent and any isomorphism $\alpha$ from $F$ onto $G$, there is a vertex $n$ of $E_{k}$ which is not in $G$ such that the function $\alpha \cup\{(m, n)\}$ is an isomorphism from $E_{k}[X \cup\{m\}]$ to $E_{k}[Y \cup\{n\}]$.

3. $E_{k}$ allows iso-extensions. 


\section{Proof:}

1. Consider any two finite disjoint sets $U$ and $V$ of vertices of $E_{k}$ with $U=\left\{u_{1}, u_{2}, \ldots, u_{\ell}\right\}$ and with $\ell \leq k$. Then we construct a vertex $n$ by choosing $\ell+1$ ones in its binary expansion in positions $u_{1}, u_{2}, \ldots, u_{\ell}$ and one position $x$ which is large enough to ensure that $x>u_{i}$ for each $i$, that $n>u_{i}$ for each $i$, and that $n$ is not a vertex of $V$; there are zeros in all the other positions of the binary expansion of $n$. Clearly, this vertex has the required properties to prove the $k$-adjoining property for $E_{k}$.

2. Consider any two finite, isomorphic, induced subgraphs $F$ and $G$ of $E_{k}$ with vertex sets $X$ and $Y$ respectively, any vertex $m$ of $E_{k}$ not in $F$ which is larger than each vertex of $F$ to which it is adjacent and any isomorphism $\alpha: X \rightarrow Y$ from $F$ onto $G$.

Consider the partition of the vertex set $X$ of $F$ into the disjoint subsets $U$ and $V$, which are, respectively, the subsets containing the vertices of $F$ adjacent to and not adjacent to $m$ in $E_{k}$. Then the required vertex $n$ of $E_{k}$ not in $Y$ can be constructed using the $k$-adjoining property of $E_{k}$ by letting $n$ be a vertex of $E_{k}$ which is adjacent to each vertex of $\alpha(U)$ and to no vertex of $\alpha(V)$. Then, clearly, $n$ is the required vertex with which we can extend the domain of the isomorphism $\alpha$ to include $m$.

3. Consider any two finite, isomorphic, induced subgraphs of $E_{k}$. The required extension of any isomorphism between them can now clearly be built through a recursive process using part 2 . of this lemma.

We remark that, in Definition 4 of the $k$-adjoining property, the existence of denumerably many vertices $n$ could have been specified with preservation of part 1 . of Lemma 3. Also, from 2. and 3. of Corollary 1 we see that, if $C$ is universal in $\mathcal{C}_{k}$ then $E_{k} \leq C$ and $C \leq E_{k}$. If the graphs involved were finite graphs, then this implies that $C$ is isomorphic to $E_{k}$. One wonders if it can be shown in this situation, with denumerable graphs, that these graphs are isomorphic too. There could well be graphs which are universal for, even in, $\mathcal{C}_{k}$ which neither allow iso-extensions nor have the $k$-adjoining property, and hence are not isomorphic to $E_{k}$.

\subsection{Universality for $k$-degenerate graphs}

We now investigate, similarly to what was done in the previous subsection, universality for $k$ degenerate graphs and start with the definitions we need.

Definition 6 [15] A finite graph $G$ is defined to be $k$-degenerate if the minimum degree $\delta(H)$ of each induced subgraph $H$ of $G$ satisfies $\delta(H) \leq k$.

There is a strong relationship between the properties of a graph $G$ "to have colouring-number at most $k+1$ " and "to be a $k$-degenerate graph" and they are, in fact, equivalent if $G$ is a finite graph. This is now recorded as

Lemma 4 A finite graph has colouring-number at most $k+1$ if and only if it is $k$-degenerate.

Proof:

Let $G$ be a finite graph with colouring-number at most $k+1$ and suppose the vertices of $G$ are labelled as $v_{1}, v_{2}, \ldots, v_{n}$ in such a way that for each positive integer $1 \leq \ell \leq n$ the degree of $v_{\ell}$ in the subgraph of $G$ induced by $\left\{v_{1}, v_{2}, \ldots, v_{\ell}\right\}$ is at most $k$. Then, for each induced subgraph $H$ of $G$, it follows that $\delta(H) \leq k$ since the vertex $v_{m}$ of $H$ with the largest label $m$ will have degree at most $k$ in $H$.

For the converse, suppose that $G$ is a finite $k$-degenerate graph of order $n$. We define a labelling $v_{1}, v_{2}, \ldots, v_{n}$ of the vertices of $G$ inductively (starting with the largest index and working downwards) by labelling any vertex of $G$ with degree at most $k$ as $v_{n}$-it exists by the definition of $k$-degenerate since $G$ is an induced subgraph of itself. Now suppose that the labels $v_{n}, v_{n-1}, \ldots, v_{m}$ have been allocated in such a way that each $v_{j}$ has degree at most $k$ in the 
subgraph induced by $v_{j}$ and the then as yet unlabelled vertices. Then, if $m>1$, we consider the subgraph induced by the as yet unlabelled vertices and choose a vertex of degree at most $k$ from it; this vertex is then labelled $v_{m-1}$. This process clearly produces the desired labelling which is needed to prove that $G$ has colouring-number at most $k+1$.

We now turn our attention to countable graphs.

Definition 7 The set of $k$-degenerate countable graphs is defined by

$\mathcal{D}_{k}=\left\{G \in \mathcal{I}_{c}\right.$ : the minimum degree of every finite induced subgraph $H$ of $G$ satisfies $\left.\delta(H) \leq k\right\}$.

Note that $\mathcal{D}_{k}$ is also an induced-hereditary set of graphs. The inclusions between $\mathcal{C}_{k}$ and $\mathcal{D}_{k}$ next deserve our attention.

Lemma $5 \mathcal{C}_{1}=\mathcal{D}_{1}$ and, for $k \geq 2$, the strict inclusions $\mathcal{C}_{k} \subset \mathcal{D}_{k} \subset \mathcal{C}_{2 k-1}$ hold.

\section{Proof:}

The inclusion $\mathcal{C}_{1} \subseteq \mathcal{D}_{1}$ follows immediately from Lemma 4 . In order to prove that $\mathcal{D}_{1} \subseteq \mathcal{C}_{1}$ too, we only need to prove, taking Theorem 1 into account, that every 1-degenerate graph is an induced subgraph of $E_{1}$. But each 1-degenerate graph is a forest consisting of countably many countable trees as its components, and $E_{1}$ consists of denumerably many clones of the denumerable tree in which every vertex has denumerable degree: This can be seen by remarking that each positive integer $p$, of which the binary expansion does not have exactly two ones, determines an induced subgraph of $E_{1}$ which is a clone of this tree and that these trees are exactly the components of $E_{1}$. Each of these trees can be viewed level by level, starting with such a $p$ on the first level, by remarking that $p$ is adjacent to the denumerably many vertices on the next level with two ones in their binary expansions, one in position $p$ and one in some position $x$ with $x>p$. The vertices on the next level adjacent to each such $x$ are then described similarly. It is clear that every one of the different tree-components of a given 1-degenerate graph $G$ is embeddable as an induced subgraph of a different component of $E_{1}$. Hence the whole $G$ is so embeddable in $E_{1}$.

If $k \geq 2$ we consider, for the first inclusion, the vertex with the largest label index of a graph with colouring-number at most $k+1$ and use it again to show that every finite induced subgraph has a vertex of degree at most $k$. The fact that it is a strict inclusion follows by remarking that a graph (as mentioned in Section 2.2, see [9]) of which every finite subgraph has colouring-number at most $k+1$, certainly is $k$-degenerate by Lemma 4 , but has colouring-number at most $2 k$ and that this result is best possible. Hence there is a graph in $\mathcal{D}_{k}$ but not in $\mathcal{C}_{k}$, since $2 k>k+1$ when $k \geq 2$.

This remark also proves the second inclusion. The fact that this inclusion is strict too follows by remarking that the complete graph $K_{2 k+1}$ has colouring-number $2 k$ but is not $k$-degenerate.

We now discuss a universal graph $F_{k}$ for $\mathcal{D}_{k}$ and its properties. It is again obtained by keeping all the vertices but restricting the choice of edges of the Rado graph $R$.

Definition 8 The graph $F_{k}$ on $\boldsymbol{N}$ has the following edges: For given positive integers $m$ and $n$ with $m<n, m$ is adjacent to $n$ if $n$ has at most $k+1$ ones in its binary expansion and has, in particular, a one in position $m$.

It is easy to see that the graph $E_{k}$ discussed in subsection 2.2 is a proper subgraph of $F_{k}$, since it has fewer edges, and that $F_{1} \subseteq F_{2} \subseteq \cdots \subseteq R$; in part 2. of Lemma 6 we shall prove more.

In our next three results we describe some properties of the set of graphs $\mathcal{D}_{k}$ and the graphs $E_{k}$ and $F_{k}$.

Theorem 2 Let $k$ be a positive integer. Then

1. $F_{k}$ is a $(k+1)$-degenerate graph, i.e., $F_{k} \in \mathcal{D}_{k+1}$.

2. $F_{k}$ is a universal graph for $\mathcal{D}_{k}$. 


\section{Proof:}

1. Consider any finite induced subgraph $H$ of $F_{k}$ and take the largest natural number $n$ which is a vertex of $H$. If there is a vertex $m$ of $H$ which is adjacent to $n$ in $H$, then $n$ has at most $k+1$ ones in its binary expansion and only those vertices corresponding to the positions of these ones can be adjacent to $n$, i.e., $n$ is of degree at most $k+1$ in $H$. But then $\delta(H) \leq k+1$ as required. 2 . We shall prove by complete induction on the cardinality of its vertex set that every countable $k$-degenerate graph $G$ is an induced subgraph of $F_{k}$. This is clearly true for a graph with only one vertex; assume that it is true for all $k$-degenerate graphs with at most $p-1$ vertices and let $G$ be a $k$-degenerate graph with $p$ vertices. Then $\delta(G) \leq k$ so that $G$ has a vertex $v$ of degree at most $k$, say it is of degree $\ell$. But then $G-v$ is a $k$-degenerate graph with $p-1$ vertices and hence $G-v$ is an induced subgraph of $F_{k}$; assume that $m_{1}, m_{2}, \ldots, m_{\ell}$ are the vertices of $F_{k}$ corresponding to the $\ell$ neighbours of $v$ in $G$. Then we construct a number $n$ by choosing $\ell$ ones in its binary expansion in positions $m_{1}, m_{2}, \ldots, m_{\ell}$ and a one in some position $x$ which is large enough to ensure that

(i) $x$ is not one of the vertices of $F_{k}$ corresponding to vertices of $G-v$;

(ii) $x>m_{i}$ for every $i$ (which also ensures that $n>m_{i}$ for every $i$ ); and

(iii) $n$ is not a vertex of $F_{k}$ corresponding to any vertex of $G-v$;

there are zeros in all the other positions of the binary expansion of $n$. Clearly, this vertex $n$ can correspond to $v$ in an isomorphism between $G$ and an induced subgraph of $F_{k}$. This completes the induction step.

Lemma 6 Let $k \geq 2$. Then

1. $E_{k}$ is a k-degenerate graph but $F_{k}$ is not.

2. $E_{k}<F_{k}$ but $F_{k} \nless E_{k}$.

3. $F_{k} \not \leq E_{k+1}$.

Proof:

1. Consider any finite induced subgraph $H$ of $E_{k}$. Then $H$ clearly has colouring-number at most $k+1$ (since $E_{k}$ has colouring-number $k+1$ by Theorem 1) and hence $\delta(H) \leq k$ by Lemma 4 , as required.

To see that $F_{k}$ is not $k$-degenerate, we need to remark that the complete graph $K_{k+2}$, which is not $k$-degenerate, can be embedded into $F_{k}$ in a recursive way: choose (similar to the proof of Lemma 2 part 1.) the vertices as the positive integers $m_{1}, m_{2}, \ldots$ by taking $m_{1} \geq 3$, constructing each further $m_{i}$ with ones in positions $m_{1}, m_{2}, \ldots, m_{i-1}$ and stopping the construction after choosing $m_{k+2}$.

2. The fact that $E_{k}<F_{k}$ follows immediately now since $E_{k} \leq F_{k}$ ( $E_{k}$ is a $k$-degenerate graph and, by Theorem $2, F_{k}$ is universal for $\mathcal{D}_{k}$ ), while by 1 . they are not isomorphic. On the other hand, $F_{k} \nless E_{k}$ since every (induced) subgraph of $E_{k}$ is $k$-degenerate but $F_{k}$ is not.

3. A graph such as the one mentioned in Section 2.2 (see [9]) of which every finite subgraph has colouring-number $k+1$ certainly is $k$-degenerate by Lemma 4 but has colouring-number $2 k$. Hence such a graph is an induced subgraph of $F_{k}$ but not of $E_{k+1}$. So $F_{k} \not \leq E_{k+1}$.

Our next result is less satisfying than the corresponding result, Corollary 1 , for graphs with colouring-number at most $k+1$.

Corollary 2 Let $k$ be a positive integer. Then

1. $F_{1}<F_{2}<\cdots<R$.

2. If $C$ is universal for $\mathcal{D}_{k+1}$ then $F_{k} \leq C$.

3. If $C$ is universal in $\mathcal{D}_{k}$ then $C \leq F_{k}$.

Proof:

1. It follows immediately from our definition of countable $k$-degenerate graphs that $\mathcal{D}_{k-1} \subseteq \mathcal{D}_{k}$ 
for every $k \geq 2$. Hence the sequence of relations $F_{1}<F_{2}<\cdots$ follow by 1 . and 2 . of Theorem 2 and the remark (already made for the graphs $E_{k}$ but equally true for the graphs $F_{k}$ ) that no isomorphism holds between any two of them. Finally, $F_{k}<R$ is true for each $k$ since $R$ is a universal graph for $\mathcal{I}_{c}$ and $F_{k}$ is not isomorphic to $R$.

2. If $C$ is universal for $\mathcal{D}_{k+1}$, then $F_{k} \leq C$ by 1 . of Theorem 2 .

3. If $C$ is universal in $\mathcal{D}_{k}$, then $C$ is $k$-degenerate too, hence $C \leq F_{k}$ by 2 . of Theorem 2 .

The following result for $(k+1)$-degenerate graphs is very similar to the corresponding result for graphs with colouring-number $k+1$, Lemma 3 . We leave the proof to the reader.

Lemma 7 The graph $F_{k}$ has the following properties:

1. $F_{k}$ has the k-adjoining property.

2. For any two finite, isomorphic, induced subgraphs $F$ and $G$ of $F_{k}$ with vertex sets $X$ and $Y$ respectively, any vertex $m$ not in $F$ which is larger than each vertex of $F$ to which it is adjacent and any isomorphism $\alpha$ from $F$ onto $G$, there is a vertex $n$ of $F_{k}$ which is not in $G$ such that the function $\alpha \cup\{(m, n)\}$ is an isomorphism from $F_{k}[X \cup\{m\}]$ to $F_{k}[Y \cup\{n\}]$.

3. $F_{k}$ allows iso-extensions.

We remark (again) that, in the definition of the $k$-adjoining property, the existence of denumerably many vertices $n$ could have been specified with preservation of part 1 . of Lemma 7 too. In the light of Lemma 4, it would be of interest to prove or to disprove that there exists a universal graph in $\mathcal{D}_{k}$ similar to $E_{k} \in \mathcal{C}_{k}$.

\section{Constructing universal graphs for general graphs}

We describe in this section uniform recursive constructions of universal graphs for inducedhereditary properties of general graphs and for products of such properties. The classes of countable graphs under consideration are general in the broadest sense: they may sport any combination of your favourite prescriptions and proscriptions of features. They may have loops or not; finitely many multiple edges between pairs of vertices or not; directed edges or undirected edges; edges or vertices (or both) labelled with labels from any prescribed finite set or not; etc.

\subsection{Constructing a universal countable graph for any induced-hereditary property}

Let $\mathcal{P}$ be any property, or class, of graphs in the above very general sense with the following characteristics:

- every member of $\mathcal{P}$ has a countable vertex set;

- $\mathcal{P}$ is an induced-hereditary graph property, i.e. every induced subgraph of a member of $\mathcal{P}$ is a member of $\mathcal{P}$ (where definitions of induced subgraphs, isomorphic graphs, etc. can be generalised from [4], where directed edges and labels are considered).

We shall now prove, by a recursive construction, that a countable graph which is universal for $\mathcal{P}$ exists. Before we do so, some notation is useful.

If $G_{1}<G_{2}<\ldots$ is a countable sequence of finite graphs with $V\left(G_{1}\right) \subseteq V\left(G_{2}\right) \subseteq \ldots$, then the limit of this sequence of graphs is the graph with vertex set $\bigcup_{i} V\left(G_{i}\right)$ (with loops, if any are in the $G_{i}$ 's) and edge set the union of the edge sets of these graphs (with their directions, labels, etc. that may be in the $G_{i}$ 's).

Let $\mathcal{F}$ be a skeleton of the class of finite members of $\mathcal{P}$ : every isomorphism class of finite members of $\mathcal{P}$ therefore has exactly one representative in the set $\mathcal{F}$. Then $\mathcal{F}$ has a countable partition

$$
\mathcal{F}=\mathcal{F}_{1} \cup \mathcal{F}_{2} \cup \ldots \cup \mathcal{F}_{n} \cup \ldots
$$


where $\mathcal{F}_{n}$ is the set of members of $\mathcal{F}$ with an $n$-element vertex set. Note that, since $\mathcal{P}$ is inducedhereditary, if $\mathcal{F}_{n+1} \neq \emptyset$, then $\mathcal{F}_{n} \neq \emptyset$; so from any non-empty level downward all levels are non-empty. $\left|\mathcal{F}_{n}\right|$ denotes the number of graphs in the $n^{\prime}$ th level set $\mathcal{F}_{n}$. Also note that $G \in \mathcal{F}_{n}$ sometimes means that $G \cong G^{\prime} \in \mathcal{F}_{n}$.

Remember that if $G \in \mathcal{F}_{n+1}$ (meaning, more precisely, that $G$ is a clone of an element of $\mathcal{F}_{n+1}$ ), then every proper induced subgraph of $G$ is (isomorphic to) an element of $\mathcal{F}_{1} \cup \mathcal{F}_{2} \cup \ldots \cup \mathcal{F}_{n}$. In particular, every induced subgraph of $G$ obtained by removing one of its vertices is in $\mathcal{F}_{n}$. Conversely, if $H \in \mathcal{F}_{n}$ and $G$ has property $\mathcal{P}$ and one more vertex than $H$, then $G \in \mathcal{F}_{n+1}$.

We shall, using this notation, construct a sequence of graphs $U_{1}, U_{2}, \ldots$ by recursion on the indices $1,2, \ldots$ such that

- $U_{1}<U_{2}<\cdots$, i.e. each is (really in this instance, not just isomorphic to) a proper induced subgraph of the next one;

- if $G \in \mathcal{F}_{1} \cup \mathcal{F}_{2} \cup \ldots \cup \mathcal{F}_{n}$, then $G<U_{n}$ (now meaning that $G$ is isomorphic to an induced subgraph of $U_{n}$ );

- the vertex set of $U_{n}$ has cardinality $\left|\mathcal{F}_{1}\right|+\left|\mathcal{F}_{2}\right|+\cdots+\left|\mathcal{F}_{n}\right|$ (since each vertex of $U_{n}$ will owe its existence to precisely one graph in $\mathcal{F}_{1} \cup \mathcal{F}_{2} \cup \ldots \cup \mathcal{F}_{n}$ );

- the limit $U$ of the sequence $U_{1}, U_{2}, \ldots$ is universal for $\mathcal{P}$.

We are now ready for the proof of

Theorem 3 Let $\mathcal{P}$ be any induced-hereditary property of countable general graphs. Then there exists a countable universal graph $U$ for $\mathcal{P}$.

\section{Proof:}

Let $U_{1}=\sqcup \mathcal{F}_{1}$, the disjoint union of the finite set of graphs (there may be more than one since loops or finite vertex labellings are allowed) in $\mathcal{F}_{1}$. Note that thus far there are no edges, except possible loops, in sight.

Suppose now that $U_{1}, U_{2}, \ldots, U_{n}$ has been constructed. Then we construct $U_{n+1}$ as follows: Suppose $\left|\mathcal{F}_{n+1}\right|=k$. Then we take $k$ new vertices $v_{1}, v_{2}, \ldots, v_{k}$ (not ocurring in $U_{n}$ ), each $v_{i}$ linked by its index $i$ to some element $G_{i}$ of $\mathcal{F}_{n+1}$. The vertex set $V\left(U_{n+1}\right)$ of $U_{n+1}$ is then defined (with preservation of vertex labels and loops on $V\left(U_{n}\right)$, if applicable) to be $V\left(U_{n+1}\right)=$ $V\left(U_{n}\right) \cup\left\{v_{1}, v_{2}, \ldots, v_{k}\right\}$.

Next we define the edge set $E\left(U_{n+1}\right)$ of $U_{n+1}$. All the edges of $U_{n}$ (with their multiplicities, directions, labels, etc. preserved, if applicable) are edges of $U_{n+1}$, i.e., $E\left(U_{n}\right) \subseteq E\left(U_{n+1}\right)$. Furthermore, in $U_{n+1}$ there are no edges between any $v_{i}$ and $v_{j}$ for $i \neq j$. Describing the new edges in $U_{n+1}$ requires some preliminary work:

Consider any fresh $v_{i} \in V\left(U_{n+1}\right)$ and its (unique) corresponding $G_{i} \in \mathcal{F}_{n+1}$ with $n+1$ vertices. Take any $v \in V\left(G_{i}\right)$. Then the induced subgraph $H_{i}^{\prime}=G_{i}-v$ of $G_{i}$ has property $\mathcal{P}$ and hence is isomorphic to some $H_{i} \in \mathcal{F}_{n}$. But, by the construction of $U_{n}$, the latter is universal for $\mathcal{F}_{n}$, and hence there exists an induced subgraph $L_{i}$ of $U_{n}$ which is isomorphic to $H_{i}$ and to $H_{i}^{\prime}=G_{i}-v$. Consider $\alpha_{i}: L_{i} \cong H_{i}^{\prime}=G_{i}-v$. Now we decorate $v_{i}$ of $U_{n+1}$ with everything that it needs for us to be able to extend $\alpha_{i}$ to $\alpha_{i}^{+}: U_{n+1}\left[L_{i} \cup\left\{v_{i}\right\}\right] \cong G_{i}$, as follows:

- Should $v$ have a vertex-label in $G_{i}$, we assign that label to $v_{i}$ in $U_{n+1}$.

- Should $v$ have a loop in $G_{i}, v_{i}$ gets a loop (perhaps labelled) in $U_{n+1}$.

- Whenever $v$ has an edge (or multiple edges) to $w$ in $G_{i}$, we prescribe an edge (or multiple edges of the same multiplicity) between $v_{i}$ and $\alpha^{-1}(w) \in V\left(U_{n}\right)$ - the latter edge(s) decorated, if applicable, with the corresponding decoration(s) from $G_{i}$.

Doing what we have just described for each $v_{i}$ and $G_{i}, i=1,2, \ldots, k$, separately, completes the construction of $U_{n+1}$ from $U_{n}$ and $\mathcal{F}_{n+1}$.

We still need to prove that for every graph $G \in \mathcal{P}$ we have that $G$ is an induced subgraph of $U$, the limit of the sequence $U_{1}, U_{2}, \ldots$ to prove that $U$ is universal for $\mathcal{P}$. Hence consider any $G \in \mathcal{P}$. 
- If $G$ is finite, say $|V(G)|=n$, then $G \cong G^{\prime} \in \mathcal{F}_{n}$ and $G^{\prime}<U_{n}<U$, so $G<U$ (since $<$ is transitive).

- If $V(G)$ is denumerable we may assume that $V(G)=\mathbf{N}$. For any positive integer $n$ we can then prove (like in the finite case) that the subgraph $G_{n}$ of $G$ induced by $[n]=\{1,2, \ldots, n\}$, which is in $\mathcal{P}$ since the latter is induced-hereditary, is an induced subgraph of $U_{n}$. Hence $G=\bigcup_{n} G_{n}<\bigcup_{n} U_{n}=U$.

It is immediate to see that $U$ is countable since each of the countably many steps in the construction of $U$ involves a finite number of new vertices.

\subsection{Constructing universal graphs for products of properties}

We start by defining, in a similar way as was done for example in [2] for finite graphs, a product of properties.

Definition 9 Let $\mathcal{P}$ and $\mathcal{Q}$ be any induced-hereditary properties of (simple, for now) countable graphs. The product of $\mathcal{P}$ and $\mathcal{Q}$, denoted by $\mathcal{P} \circ \mathcal{Q}$, is the property consisting of all (countable) graphs $G$ for which there is a partition of the vertex set $V(G)$ into two parts $X$ and $Y$ such that the subgraph $G[X]$ of $G$ induced by $X$ is in $\mathcal{P}$ and the subgraph $G[Y]$ of $G$ induced by $Y$ is in $\mathcal{Q}$.

We remark that this definition can clearly be extended to products of more than two properties of graphs and that these products are induced-hereditary properties of countable graphs. By the construction in the previous subsection, there is a countable universal graph $U(\mathcal{P} \circ \mathcal{Q})$ for $\mathcal{P} \circ \mathcal{Q}$.

The question arises whether the universal graphs $U(\mathcal{P})$ and $U(\mathcal{Q})$ (for $\mathcal{P}$ and $\mathcal{Q}$ separately) can somehow be employed in the construction of another universal graph, say $W(\mathcal{P} \circ \mathcal{Q})$, for $\mathcal{P} \circ \mathcal{Q}$. We now describe such a construction which gives a universal graph for the finite graphs in $\mathcal{P} \circ \mathcal{Q}$ and denote the graph we construct by $W$ for short.

Besides $U(\mathcal{P})$, we need a denumerable set of pairwise disjoint, triply indexed clones of $U(\mathcal{Q})$,

$$
U(\mathcal{Q})_{i j k}, i j k \in \mathbf{N}^{3} \text {, with each } U(\mathcal{Q})_{i j k} \cong U(\mathcal{Q}) .
$$

The role of the three indices will become clear as we proceed. Every $U(\mathcal{Q})_{i j k}$ is also disjoint from $U(\mathcal{P})$.

We are now ready to describe the vertex set, $V(W)$, of $W$ :

$$
V(W)=V(U(\mathcal{P})) \sqcup V\left(\sqcup U(\mathcal{Q})_{i j k}\right),
$$

where $V\left(\sqcup U(\mathcal{Q})_{i j k}\right)=\sqcup_{i j k} V\left(U(\mathcal{Q})_{i j k}\right)$ is the disjoint union of the vertex sets of the different $U(\mathcal{Q})_{i j k}$.

We now begin the description of the edge set of $W$ :

$$
E(W) \supset E(U(\mathcal{P})) \cup E\left(\sqcup U(\mathcal{Q})_{i j k}\right),
$$

i.e., all the edges of $U(\mathcal{P})$ and of all the copies of $U(\mathcal{Q})$ are edges of $W$ - but there are more edges in $W$. To describe these extra edges in $W$ we shall use the index-triples $i j k$, but we also need some more notation. Let

$$
\mathcal{F}(\mathcal{P})=\left\{G_{1}, G_{2}, \ldots\right\}
$$

be any enumeration of the (countable, most likely denumerable) skeleton of the class of finite members of $\mathcal{P}$ used in the construction of $U(\mathcal{P})$. Each element $G_{i}$ of $\mathcal{F}(\mathcal{P})$ has a canonical occurrence as (a clone of) an induced subgraph of $U(\mathcal{P})$. Similarly, for $\mathcal{Q}$ we have

$$
\mathcal{F}(\mathcal{Q})=\left\{H_{1}, H_{2}, \ldots\right\}
$$


with $H_{i}$ occurring canonically in $U(\mathcal{Q})$. The canonical clone of $G_{i}$ in $U(\mathcal{P})$ or of $H_{j}$ in $U(\mathcal{Q})$ or in $U(\mathcal{Q})_{i j k}$ will be denoted by the same symbol, $G_{i}$ or $H_{j}$.

When $G_{i} \in \mathcal{F}(\mathcal{P})$ has $m$ vertices and $H_{j} \in \mathcal{F}(\mathcal{Q})$ has $n$ vertices, then there are $m n$ potential edges between vertices of $G_{i}$ and vertices of $H_{j}$. Hence there are $2^{m n}$ different potential sets of edges between the vertices of $G_{i}$ and the vertices of $H_{j}$. Each of these possibilities represents a finite graph in $\mathcal{P} \circ \mathcal{Q}$ (not all of them necessarily non-isomorphic).

Now $U(\mathcal{Q})_{i j 1}, U(\mathcal{Q})_{i j 2}, \ldots, U(\mathcal{Q})_{i j 2^{m n}}$ are $2^{m n}$ clones of $U(\mathcal{Q})$. We can write $U(\mathcal{Q})_{i j k}, 1 \leq k \leq$ $2^{m n}$, implying that within $U(\mathcal{P})$ we have $\left|V\left(G_{i}\right)\right|=m$ and within $U(\mathcal{Q})$ we have $\left|V\left(H_{j}\right)\right|=n$. In $W$ we now add the following edges: For each $G_{i} \in \mathcal{F}(\mathcal{P})$ and each $H_{j} \in \mathcal{F}(\mathcal{Q})$ and each possibility $k$ of the $2^{m n}$ possibilities of edge sets between $V\left(G_{i}\right)$ and $V\left(H_{j}\right)$ we add to the edges of $W$ those edges between $V(U(\mathcal{P}))$ and $V\left(U(\mathcal{Q})_{i j k}\right)$ which correspond to the $k$ 'th set of edges between $V\left(G_{i}\right)$ (in $V(U(\mathcal{P}))$ ) and $V\left(H_{j}\right)$ (in $V\left(U(\mathcal{Q})_{i j k}\right)$ ). That completes the description of the edge set of $W$. Each finite graph in $\mathcal{P} \circ \mathcal{Q}$ has now a canonical occurrence in $W$, and the following theorem holds.

Theorem $4 W(\mathcal{P} \circ \mathcal{Q})$ is universal for $(\mathcal{P} \circ \mathcal{Q})_{f}$.

What can we say about a possible universal graph for the denumerable ones? In Lemma 1 we saw how playing with disjoint unions of denumerable graphs tends to propel us to the dizzy heights of uncountability. May it help if $\mathcal{P}$ and $\mathcal{Q}$ are of finite character?

\section{Concluding remarks: Contrasting the two constructions}

We have now encountered two approaches, styles of constructing universal graphs in and for induced-hereditary graph properties:

A: the construction of a universal graph $U$ in some properties $\mathcal{P}$ as found in, e.g., [3], [4], [17] and [18], and Sections 2.2 and 2.3 of this paper.

B: the construction of a universal graph $U$ for any induced-hereditary property $\mathcal{P}$ as found in Section 3 of this paper.

Some contrasts between A and B are salient:

(a) Symmetry: A-constructed universal graphs have extremely large automorphism groups. For instance: $R$ has $2^{\aleph_{0}}$ conjugacy classes of cyclic automorphisms, i.e., automorphisms for which the vertices of $R$ can be labelled by the set of integers $\mathbf{Z}$ so that the automorphism is the cyclic shift $x \mapsto x+1$ ([5], slide 18). But one imagines that B-constructed ones have, relatively speaking, very small automorphism groups.

(b) Thrift: Related to their great symmetry, A-constructed universal graphs are prodigal (as elaborated on as "abundance" in [3]), while B-constructed universal graphs are frugal in comparison, missing the "non-locality" and ubiquity of an extension or adjoining property or homogeneity. An A-constructed homogeneous universal graph "looks the same" from the viewpoint of any of its vertices. This is so since, by homogeneity, any two isomorphic finite induced subgraphs, and in particular any two vertices, can be interchanged by an automorphism of the whole graph. Intuitively speaking, you can "pick up" the graph by any of its vertices, "shake it out", and "downward" from that vertex it unfolds all the way in exactly the same pattern. For A-constructed universal graphs which are not homogeneous, but have the somewhat weaker property of allowing iso-extensions (like $E_{k}$ and $F_{k}$ in Section 2), any two vertices (or finite isomorphic induced subgraphs) still have isomorphic induced denumerable supergraphs within the graph. By contrast, in a B-constructed $U$ for $\mathcal{P}$, every finite graph in $\mathcal{P}$ has one "canonical" occurrence within $U$, apart from possible other accidental occurrences. This canonical occurrence is even anchored to the specific vertex of $U$ for the existence of which it is responsible.

(c) Definition: In A the universal graph is established in Rado style by fiat, a definition need- 
ing an argument to show that it harbours every graph from $\mathcal{P}$. In $\mathrm{B}$ the universal graph is built incrementally in recursive steps, showing directly how the elements of $\mathcal{P}$ of growing size are incorporated. The induced finite subgraph $U_{n}$ of the universal graph $U$ accommodates all those graphs in $\mathcal{P}$ with no more than $n$ vertices. It is not immediately clear whether anything similar can be explicated elegantly for an A-constructed universal graph. For instance, can one easily define a finite induced subgraph of $R$ which accommodates (maybe even tightly) all simple graphs with at most $n$ vertices?

(d) Features of hereditary properties: It seems intuitively as if B may be more illuminating than A in the study of certain features such as the "speed" and the "boundedness of clique-width" (as discussed in [1]) for induced-hereditary properties. A similar remark holds with respect to finding universal graphs with a minimum number of vertices for a finite number of finite graphs (as discussed in [16]). We remark that, when few vertices is the thrifty issue, the recursive step constructing $U_{n+1}$ from $U_{n}$ in the proof of Theorem 3 can be made more parsimonious: Add a new vertex $v_{i}$ to $U_{n}$ only for those $G_{i} \in \mathcal{F}_{n+1}$ which are not, accidentally, already isomorphic to some induced subgraph of $U_{n}$.

(e) Generality: Constructions of stripe A deliver a universal graph in $\mathcal{P}$. Some inducedhereditary properties $\mathcal{P}$ do not contain universal graphs, as seen in the table in Section 2.1. B always creates a universal graph for $\mathcal{P}$.

(f) Complexity: For a given $\mathcal{P}$ there is the question of the computational complexity of generating the B-sequence $U_{1}, U_{2}, \ldots$ No analogous question for $\mathrm{A}$ springs to mind.

\section{Acknowledgement}

We express our indebtedness and gratitude to the reviewer for the journal who spotted a mistake in an earlier version of this article.

\section{References}

[1] P. Allen, V. Lozin and M. Rao, Clique-width and the speed of hereditary properties, The Electronic Journal of Combinatorics 16 (2009), 1 - 11.

[2] M. Borowiecki, I. Broere, M. Frick, G. Semanišin and P. Mihók, A survey of hereditary properties of graphs, Discussiones Mathematicae Graph Theory 17 (1997), 2 - 38.

[3] I. Broere and J. Heidema, Constructing an abundance of Rado graphs, Manuscript, submitted for publication, January 2010.

[4] I. Broere and J. Heidema, Some universal directed labelled graphs, Manuscript, submitted for publication, March 2010.

[5] P.J. Cameron, The random graph revisited, Australian Mathematical Society winter meeting in Brisbane, and European Congress of Mathematics in Barcelona, July 2000. http://www.maths.qmw.ac.uk/ pjc/slides/rgr.pdf

[6] G. Cherlin and P. Komjáth, There is no universal countable pentagon-free graph, Journal of Graph Theory 18 (1994), 337 - 341.

[7] G. Cherlin and N. Shi, Graphs omitting a finite set of cycles, Journal of Graph Theory 21 (1996), $351-355$.

[8] R. Diestel, Graph theory, Electronic Edition, Springer-Verlag, Heidelberg, 2005. Graduate Texts in Mathematics, Vol. 173. 
[9] P. Erdős and A. Hajnal, On chromatic number of graphs and set-systems, Acta Math. Hungar. 17 (1966), $61-99$.

[10] R. Fraïssé, Sur l'extension aux relations de quelques propriétiés connues des ordres, C. R. Acad. Sci. Paris 237 (1953), 508 - 510.

[11] Z. Füredi and P. Komjáth, On the existence of countable universal graphs, Journal of Graph Theory 25 (1997), $53-58$.

[12] A. Hajnal and J. Pach, Monochromatic paths in infinite coloured graphs, in Colloquia Mathematica Societatis János Bolyai 37, Finite and infinite sets, Eger (Hungary) (1981), $359-369$.

[13] C.W. Henson, A family of countable homogeneous graphs, Pacific J. Math. 38 (1971), 69 83.

[14] P. Komjáth and J. Pach, Universal graphs without large bipartite subgraphs, Mathematika 31 (1984), $282-290$.

[15] R. Lick and A.T. White, k-degenerate graphs, Canad. J. Mathematics, 22 (1970), 10821096.

[16] V. Lozin and G. Rudolf, Minimal universal bipartite graphs, Ars Combinatoria 84 (2007), $345-356$.

[17] P. Mihók, J. Miškuf and G. Semanišin, On universal graphs for hom-properties, Discussiones Mathematicae Graph Theory 29 (2009), no. 2, 401 - 409.

[18] R. Rado, Universal graphs and universal functions, Acta Arith. 9 (1964), 331 - 340. 